\title{
Depth separation and the Ponzo illusion
}

\author{
ROBERT PATTERSON and ROBERT FOX \\ Vanderbilt University, Nashville, Tennessee
}

\begin{abstract}
The Ponzo illusion refers to an apparent change in length of objectively equal parallel lines induced by enclosure within an acute angle. The present study investigated this illusory change in stimulus extent as a function of the relative depth positions of the parallel lines and the inducing angle. To permit facile and unconfounded manipulation of apparent depth, the stimuli comprising the Ponzo configuration were stereoscopic contours formed from dynamic random. element stereograms. The main results were: (1) apparent depth separation exerted a strong influence on illusion magnitude; (2) this influence was asymmetrical in that illusion magnitude decreased when the inducing angle appeared in depth behind the parallel lines and increased when the inducing angle appeared in depth in front of the lines. These data are consistent with a general theory of space perception that assumes that information about depth position is processed prior to information about stimulus characteristics.
\end{abstract}

Of the many visual illusions that have been subjected to intensive scrutiny, one of the most popular is the distortion in line length that characterizes the Ponzo illusion, illustrated in Figure 1. While of interest in its own right, the illusion is used here as a convenient way of investigating the effect of position in depth on stimulus interaction. Operationally, the present inquiry poses the following question: Would the magnitude of the illusion be altered if the inducing figure (acute angle) occupied a depth position different from that of the test figure (parallel lines)? That question is provoked theoretically by the implications it has for general theories of visual space perception and empirically by data indicating that depth position can exert a very significant influence on certain interactive visual phenomena. Specifically, our group has found an effect of depth position on visual masking (Lehmkuhle \& Fox, 1980) and on lateral interference (Fox \& Patterson, 1981). In both studies, masking and interference declined as the difference in depth position of the interacting forms increased, when the test form appeared to be in a depth plane in front of the masking form and, thus, closer to the observer. Yet, when depth positions were reversed, thereby placing the masking form in front of the test, the strength of the masking and interference effects tended to increase. While an effect of depth position was anticipated on theoretical grounds, the asymmetry of its position was unexpected. This asymmetry was called the "front effect" by Lehmkuhle and Fox (1980), who hypothesized that it might represent an intrinsic positive bias of the visual system

Support for this investigation was provided by Grants EY 00590-17, from the National Institutes of Health, and N0001476-C-1101, from the Office of Naval Research. Requests for reprints should be sent to either author, Department of Psychology, Vanderbilt University, Nashville, Tennessee $\mathbf{3 7 2 4 0 .}$ for the stimulus that occupies the depth plane closest to the observer.

The purpose of this study was to determine if depth position would produce similar effects on the magnitude of the Ponzo illusion, which is characterized by an apparent change in stimulus extent rather than by an impairment in perceptability. Note that it is possible to regard the interactions associated with masking and lateral interference as destructive or inhibitory (e.g., threshold elevation), while the interactions of many visual illusions can be thought of as distortive, a distinction that may reflect a difference in mechanism. Before describing the present study, consideration should be given to several prior studies, undertaken for various reasons, that have some bearing on the question of the effect of depth position on the Ponzo illusion.

Green, Lawson, and Godek (1972) presented the illusion as a stereogram consisting of discrete contours. They found that illusion magnitude diminished when the test lines, with crossed disparity, appeared in depth planes in front of the inducing angle, yet increased with uncrossed disparity that placed the lines in depth planes behind the angle. They attributed this asymmetrical effect in depth position to changes in the apparent size of the test lines induced by size constancy. That is, when the lines were presented with crossed disparity, they appeared smaller, thereby increasing the apparent spacing between them and the edges of the angle. Conversely, when the lines were presented with uncrossed disparity, they appeared larger, thereby decreasing the apparent spacing between them and the edges of the angle. This would account for the asymmetrical pattern that was found, assuming that illusion magnitude varies inversely with such spacing.

Yet this asymmetry is not consistent with the adjacency principle developed by Gogel (e.g., Gogel, 
1978), which would posit a symmetrical decrease in illusion magnitude as the difference in depth position between interacting stimulus elements increases. Motivated by this departure from the adjacency principle, Gogel (1975) used a combination of absolute distances cues and stereoscopic depth cues to manipulate the apparent depth position of the elements of the Ponzo illusion. In one condition, in which a single angle was used, the effect of depth position on illusion magnitude was similar to that observed by Green et al. (1972). In a second condition, two inducing angles were located in different depth planes with their apexes oriented in opposite directions. This yielded a more complex pattern of results that Gogel could interpret as being generally consistent with the adjacency principle. No compelling explanation could be advanced, however, for the failure of the results to conform to the symmetrical relationship predicted by the adjacency principle when a single inducing angle was used. Gogel suggests several possibilities, including differential effects of attention and conflicting information about depth induced by the interaction of absolute and relative depth cues. Although it is not clear why the single-inducing-angle condition produced an asymmetrical effect of depth position while the two-angle condition produced a symmetrical effect, the most important aspect of these results is that, in both cases, depth position exerted a significant influence on illusion magnitude.

In a brief report, Hennessy and Leibowitz (1972) used a method of physical separation (lines on a glass sheet) to locate the test lines of the illusion at a depth position in front of the inducing angle. They found a reduction in illusion magnitude under the condition of depth separation.

Finally, Julesz (1971) presents a static randomelement stereogram of the Ponzo illusion in which the angle and test lines are separated in depth. He notes, anecdotally, that the depth separation appears to change illusion magnitude. Yet, a more rigorous check on this observation, made in this laboratory, did not yield general agreement. ${ }^{1}$

Together, the results of these studies are not unequivocal. One factor responsible for this disagreement is the difficulty encountered in manipulating the apparent depth of stimuli without introducing confounding changes in proximal stimulation. Typically, only a limited range of depth positions can be varied, and it is difficult to compensate for the change in apparent size that covaries with the change in apparent depth. In general, these restrictions on experimental manipulations have thwarted research on the effect of depth position on stimulus interaction.

One technique that side-steps the problem of proximal stimulation, yet allows for large changes in depth, is the use of stereoscopic stimuli generated by random-element stereograms (e.g., Julesz, 1971).
Such stimuli arise only at central stages of the visual system; nevertheless, they can induce many of the same perceptual phenomena (e.g., aftereffects, illusions, masking) as physical stimuli. These features were exploited in this study by presenting the elements that comprise the Ponzo illusion as stereoscopic stimuli.

\section{METHOD}

\section{Subjects}

Twelve persons, paid for their services and recruited from the student population at Vanderbilt, served as subjects. All possessed normal visual capacities and had had prior experience in perceiving stereoscopic contours formed from dynamic random-element stereograms. They were, however, unaware of the hypothesis under test.

\section{Apparatus}

Only a brief description of the system for generating dynamic random-element stereograms need be given here, since more complete descriptions are given in Fox and Patterson (1981), Lehmkuhle and Fox (1980), and Shetty, Brodersen, and Fox (1979). For the present study, three components comprised the system: the display, the electronic control unit, and the optical programmer.

The display was a 19-in. commercial-grade color television receiver, modified so that random-dot matrices consisting of thousands of red and green dots could be displayed continuously and replaced at random 60 times per second. When an observer views this display with appropriate red and green filters before the eyes, the red and green dots are physically segregated to the separate eyes, thereby fulfilling the requirements of stereoscopic presentation (i.e., the anaglyph method of stereoscopic presentation).

The electronic unit generated the random-dot matrices and provided control over all significant parameters of the stereoscopic display. These included disparity direction and magnitude, exposure duration, and $x / y$ position of the stereoscopic forms. The continuous generation and replacement of dots produced an apparent motion similar to that observable on an untuned TV channel, yet such motion did not impair perceptibility of the stereoscopic forms.

The optical programming device, of which two were used, consists of a video camera modified to act as a flying spot scanner. Any achromatic physical configuration that is scanned or viewed by the scanner is convertible to an equivalent stereoscopic configuration. This is accomplished by using signals from the scanner to define those $x / y$ positions in any given video frame where disparity is to be inserted to produce a stereoscopic form. In the present study, one programmer scanned a high-contrast drawing of two horizontal lines of equal length that served, in the stereoscopic mode, as the basis for the test lines of the Ponzo illusion. The second scanner viewed a similar drawing of an acute angle that provided, in the stereoscopic mode, the basis for the inducing angle of the Ponzo illusion. The dimensions of these elements that comprised the illusion are given in Figure 1.

\section{Design and Procedure}

To quantify the illusion, which consists of an apparent difference in the length of the objectively equal parallel test lines, the subjects were required to make estimates of the length of one line relative to the other. Half of the subjects were instructed to regard the lower line as being 10 arbitrary units in length and then, given that standard, were asked to make estimates of the apparent length of the upper line. The other half of the subjects were given similar instructions, except that the upper line was given as the standard of 10 units in length. Judgments were made with subjects seated at a fixed distance from the screen $(150 \mathrm{~cm})$ with the head stabilized by a head- and chinrest. Five judgments were 
obtained from each subject under each of six conditions. For all conditions, the test lines remained at the same position in depth, appearing in front of the display with a crossed disparity of $22^{\prime} 0^{\prime \prime}$ '. In one condition, the control condition, the test lines were presented unaccompanied by the illusion-inducing angle. In the

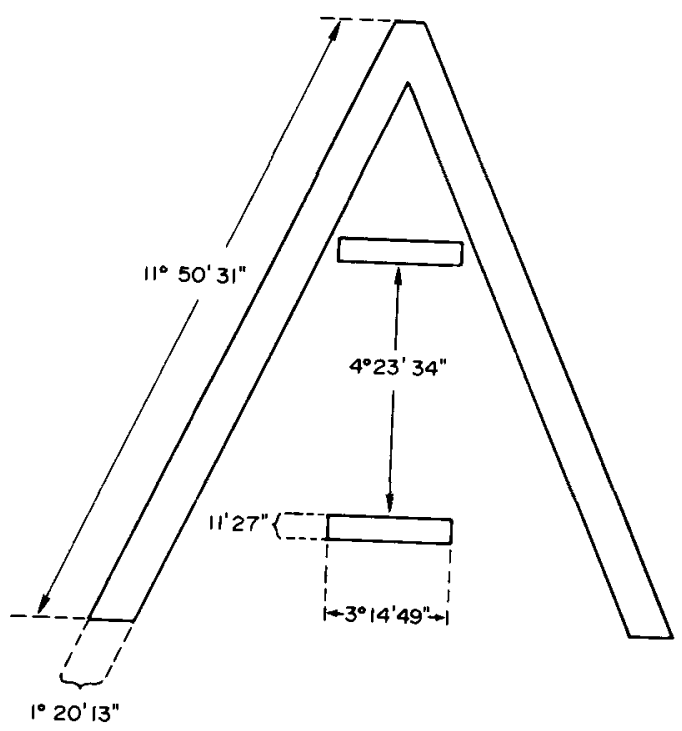

Figure 1. Configuration and dimensions of the stereoscopic test lines and inducing angle as they appeared on the display.

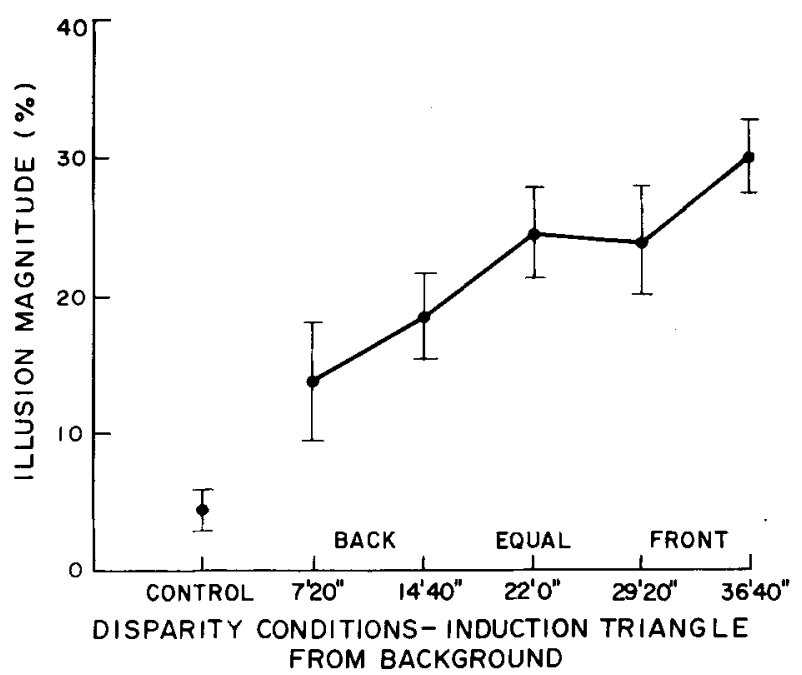

Figure 2. Illusion magnitude (percent) for the control condition, in which the test lines were presented without the inducing angle, and for five disparity conditions, in which the test lines and inducing angle were presented together. "Back," "front," and "equal" indicate the following respectively: For disparity conditions $7^{\prime} 20^{\prime \prime}$ and $14^{\prime} 40^{\prime \prime}$ (the back conditions), the inducing angle was positioned in depth behind the test lines; for disparity conditions $29^{\prime} 20^{\prime \prime}$ and $36^{\prime} 40^{\prime \prime}$ (the front conditions), the angle was positioned in depth in front of the test lines; and for disparity condition $22^{\prime} 0^{\prime \prime}$ (the equal depth condition), both the test lines and angle occupied the same depth plane. five conditions where the inducing angle was present, the angle was located at one of five depth positions: In the equal depth condition, the angle was located in the same depth plane as the test lines; in the "back" conditions, it was located at 7'20" disparity and at $14^{\prime} 40^{\prime \prime}$ disparity, thereby placing the angle at a depth position behind the test lines and further from the observer; in the "front" conditions, the inducing angle was located at disparity values $29^{\prime} 20^{\prime \prime}$ and $36^{\prime} 40^{\prime \prime}$, thereby placing it in front of the test lines and closer to the observer. Variations in the apparent width of the inducing angle, brought about by the operation of size constancy, were compensated so that the apparent width of the angle remained the same under all conditions. The 30 trials ( 5 replications $\times 6$ conditions) were administered in random orders to each subject. The subjects were given unlimited time to respond, but they usually responded within $10 \mathrm{sec}$. All data were collected in one 45-min session per subject.

\section{RESULTS}

To compute the illusion present in the five disparity conditions, the estimate of line length given by each subject under the control condition was used as a baseline. The difference between the estimate made under the disparity conditions and the control condition value was divided by the latter and multiplied by 100 to give the measure of illusion magnitude (percent). ${ }^{2}$ To compute the illusion present under the control condition, an assumed value of 10 units was used as a baseline. In this case, the difference between the estimate of line length made under the control condition and the assumed baseline of 10 units was divided by the latter and multiplied by 100 .

These mean percentage values for the five disparity conditions and for the control are shown in Figure 2. Based on results from studies that have shown an asymmetrical effect of depth position on masking (Lehmkuhle \& Fox, 1980) and on lateral interference (Fox \& Patterson, 1981), the first analysis of these data consisted of an a priori test for linear trend applied to the five disparity conditions. A significant linear trend was found $[F(1,11)=16.1, p<.01] .^{3}$ Next, the data from all six conditions were subjected to an analysis of variance (ANOVA) for randomized block designs after the sums of squares and degrees of freedom corresponding to the linear component were removed. This analysis revealed that the effect of disparity condition on illusion magnitude was significant $[F(4,55)=7.9, p<.001]$. To determine whether other trend components were present in the data from the five disparity conditions, an a posteriori test for quadratic trend was computed; there was no significant quadratic component in these data $(F<$ 1.0). Since inspection of the data revealed that the absence of other orthogonal trend components would be quite unlikely, no further trend analyses were performed.

The difference between the control condition and zero percent illusion was evaluated by a t test, which revealed that the control condition differed significantly from zero $(t=2.39, p<.05)$. 


\section{DISCUSSION}

The main feature of these results is that depth position exerts a significant effect on illusion magnitude, yet that effect is asymmetrical. When the inducing angle is to the rear of the test lines, thereby placing those lines in front of the angle, illusion magnitude declines; yet, when those relative depth positions are reversed, and the inducing angle is in front of the test lines, illusion magnitude increases. This is the same pattern of results found by Gogel (1975) in his single-inducing-angle experiment and by Green et al. (1972). It is also consistent with the study by Hennessy and Leibowitz (1972), who used a singledepth condition.

Yet, the explanation proposed by Green et al., based on changes in apparent size, is not applicable to the present data, since apparent size was kept constant. Similarly, Gogel's suggestion that the asymmetry may be due to some conflict between absolute and stereoscopic depth cues may not be applicable here, since there appears to be no grounds for a cue conflict in the present experiment.

An alternative explanation is that the asymmetry is still another manifestation of the front effect, which has previously been found for visual masking (Lehmkuhle \& Fox, 1980) and for lateral interference (Fox \& Patterson, 1981). On this view, when there are stimuli in the visual field that are spatially adjacent in the $x / y$ plane or, in equivalent terms, occupy similar visual directions, the visual system will manifest a positive bias for the stimulus that is in front of all others and closest to the observer. Within this theoretical framework, the present data suggest that the front effect is not confined to those situations involving destructive or inhibitory interaction among stimuli; it can also apply to suprathreshold interaction of the kind typified by the Ponzo illusion and similar kinds of two-dimensional visual illusions of linear extent.

Yet, quite apart from the theoretical interpretation of the asymmetry that defines the front effect, its existence implies that the depth position of stimuli plays a significant role in governing their interactions. This means that models developed to account for such interactions in two dimensions, as for example those based on lateral inhibition, are at the very least incomplete. Furthermore, it is antithetic to the general assumption that depth information is processed at a relatively late stage in the visual system, after stimuli have been analyzed and the cues to depth extracted. Rather, the significant role played by depth position is consistent with the view that depth information is processed quite early in the system and governs subsequent processing of stimuli. This, of course, is not unlike the view envisioned by the classic Gestalt psychologists (e.g., Koffka, 1935) or by contemporary formulations such as the adjacency principle enunciated by Gogel (e.g., 1978).

\section{REFERENCE NOTE}

1. Fox, R., \& Patterson, R. Effect of depth separation on the Ponzo illusion (Tech. Rep. N14-1101 81C-0001). Arlington, Va: Engineering Psychology Programs, Office of Naval Research, 1981.

\section{REFERENCES}

Fox, R., \& Patterson, R. Depth separation and lateral interference. Perception \& Psychophysics, 1981, 30, 513-520.

Goget, W. C. Depth adjacency and the Ponzo illusion. Perception \& Psychophysics, 1975, 17, 125-132.

Gogel, W. C. The adjacency principle in visual perception. Scientific American, 1978, 238, 5, 126-139.

Green, R. T., Lawson, R. B., \& Godek, C. L. The Ponzo illusion in stereoscopic space. Journal of Experimental Psychology, 1972, 95, 358-364.

Hennessy, R. T., \& Leibowitz, H. W. Perceived vs retinal relationships in the Ponzo illusion. Psychonomic Science, 1972, 28, 111-112.

JuLESz, B. Foundations of cyclopean perception. Chicago: University of Chicago Press, 1971.

KoffKa, K. Principles of Gestalt psychology. New York: HarcourtBrace, 1935.

LeHMKUhle, S., \& Fox, R. The effect of depth separation on metacontrast masking. Journal of Experimental Psychology: Human Perception and Performance, 1980, 6, 605-621.

Shetry, S. S., Brodersen, A. J., \& Fox, R. System for generating dynamic random-element stereograms. Behavior $R e-$ search Methods \& Instrumentation, 1979, 11, 485-490.

\section{NOTES}

1. In this demonstration, Julesz (1971) presents two static stereograms of the Ponzo illusion. In one stereogram, both the test lines and inducing angle appear in the same depth plane (his Figure 7.2-3), while in the other the test lines appear in depth behind the inducing angle (his Figure 7.2-4). We presented these same stereograms to 10 naive observers, who were required to make forced-choice judgments of the direction of change in illusion magnitude. Of these 10,5 reported an increase and 5 a decrease in illusion magnitude following separation in depth of the test lines and angle.

2. This method of computing illusion magnitude corrects for any residual illusion that might be present in the absence of the inducing angle. Another method of computing illusion magnitude that does not correct for this possibility is to use the standard of 10 units as a baseline. In this method, the difference between the estimate of line length given by each subject under the disparity conditions and the value of 10 is divided by the latter and multiplied by 100 . Based on this method, the mean percentage values for the $7^{\prime} 20^{\prime \prime}, 14^{\prime} 40^{\prime \prime}, 22^{\prime} 0^{\prime \prime}, 29^{\prime} 20^{\prime \prime}$, and $36^{\prime} 40^{\prime \prime}$ conditions, respectively, are: $18.1,21.7,27.8,27.4$, and 33.0 .

3. These data have been analyzed previously using an analysis of variance and the Newman-Keuls test for post hoc comparisons (see Fox \& Patterson, Note 1). The Newman-Keuls test revealed that although the decrease in illusion magnitude obtained under the $7^{\prime} 20^{\prime \prime}$ condition relative to that obtained under the $22^{\prime} 0^{\prime \prime}$ condition was statistically significant, the increase in illusion magnitude obtained under the $36^{\prime} 40^{\prime \prime}$ condition was not. A more powerful analysis of these data, however, using an a priori test of linear trend, revealed that a significant linear component is present in these data. This latter result indicates that the increase in illusion magnitude obtained under the $36^{\prime} 40^{\prime \prime}$ condition is statistically significant.

(Manuscript received September 27, 1982; revision accepted for publication March 11, 1983.) 